\title{
SYNTHESIZING SMOS ZERO-BASELINES WITH AQUARIUS BRIGHTNESS TEMPERATURE SIMULATOR
}

\author{
A. Colliander ${ }^{1}$, E. Dinnat ${ }^{2}$, D. Le Vine ${ }^{3}$, J. Kainulainen ${ }^{4}$ \\ 1) Jet Propulsion Laboratory, California Institute of Technology, \\ 4800 Oak Grove Drive, Pasadena, CA91109, USA \\ 2) Chapman University, NASA-GSFC, Greenbelt Rd, Greenbelt, MD20771 \\ 3) NASA-GSFC, Greenbelt Rd, Greenbelt, MD20771 \\ 4) Department of Radio Science and Engineering, Aalto University School of Electrical Engineering, \\ P.O.BOX 13000, FI-00076 AALTO, Finland
}

\section{INTRODUCTION}

SMOS [1] and Aquarius [2] are ESA and NASA missions, respectively, to make L-band measurements from the Low Earth Orbit. SMOS makes passive measurements whereas Aquarius measures both passive and active. SMOS was launched in November 2009 and Aquarius in June 2011.The scientific objectives of the missions are overlapping: both missions aim at mapping the global Sea Surface Salinity (SSS). Additionally, SMOS mission produces soil moisture product (however, Aquarius data will eventually be used for retrieving soil moisture too). The consistency of the brightness temperature observations made by the two instruments is essential for longterm studies of SSS and soil moisture.

For resolving the consistency, the calibration of the instruments is the key. The basis of the SMOS brightness temperature level is the measurements performed with the so-called zero-baselines [3]; SMOS employs an interferometric measurement technique which forms a brightness temperature image from several baselines constructed by combination of multiple receivers in an array; zero-length baseline defines the overall brightness temperature level. The basis of the Aquarius brightness temperature level is resolved from the brightness temperature simulator combined with ancillary data such as antenna patterns and environmental models [4]. Consistency between the SMOS zero-baseline measurements and the simulator output would provide a robust basis for establishing the overall comparability of the missions. 


\section{METHODS AND MATERIALS}

The footprints of the SMOS zero-baseline and the beams of the Aquarius are fundamentally different. This is due to the fact that the SMOS zero-baseline is designed to measure the average brightness temperature of the entire SMOS scene and, therefore, the 3-dB footprint spans over $1000 \mathrm{~km}$ in the cross-track direction and includes a piece of sky in the along-track direction (see Figure 1), whereas Aquarius is a real aperture pushbroom system which has footprints of size about $70 \mathrm{~km}$ to $150 \mathrm{~km}$ on the surface of the Earth. Although the primary purpose of the Aquarius simulator is to simulate antenna temperature corresponding to Aquarius footprints, it is able to determine the brightness temperature of a wide beam such as that of SMOS zero-baseline, since it includes the simulation of the side and back lobes of the Aquarius antenna for accuracy. Accordingly, the simulator incorporates correction for full view angle and has the capability to simulate brightness temperature emitted by sea, land, atmosphere, microwave background and celestial objects. The comparisons of this study will be made over ocean where the model is the most accurate (and which is most relevant for the SSS retrieval of the missions) and the largest contribution comes from the sea with partial effect from the sky.

The ability of the simulator to predict correct brightness temperature level for the SMOS zero-baseline has been verified. The Aquarius antenna pattern was replaced by the antenna pattern of a zero-baseline element of SMOS in the simulator. The level of the resulted antenna temperature is very close to an actual zero-baseline measurement. Figure 2 shows the simulated (left) and measured values (right). The simulation uses the middle beam of Aquarius which has incidence angle closest to the boresight incidence angle of the SMOS zero-baseline (but not the same). The location on the Earth, the pointing direction with respect to Earth and the orientation of the measurement with respect to sun and galaxy are different in these two cases.

Full simulations of the SMOS zero-baseline observations are being carried out with the actual measurement geometry. The simulations focus on observations over Pacific Ocean where the surface conditions are climatologically constant, the full field of view is as free as possible from the effect of land, and the interference from galaxy and the sun is minimal (but the significance of these interference sources will also be studied). The simulation include all observations carried out since the end of the SMOS commissioning phase which yields a data set of over 1.5 years long. This allows investigation of seasonally driven anomalies in addition to short term and instantaneous effects. 

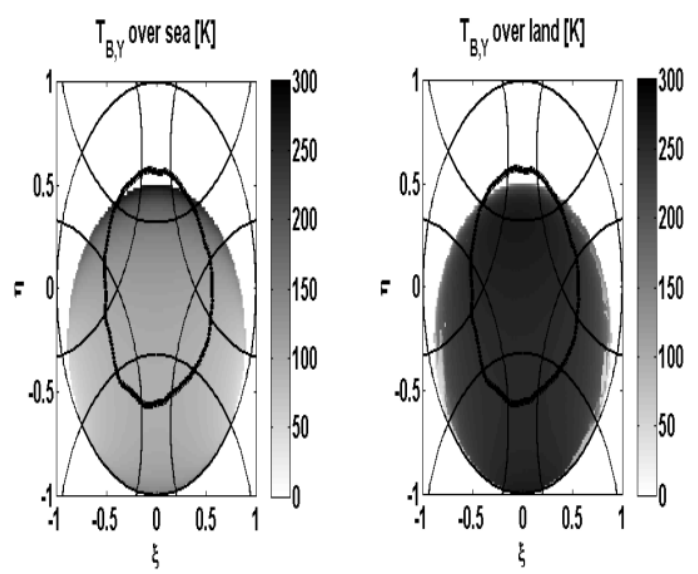

Figure 1. SMOS field of view and the 3-dB footprint of a zero-baseline radiometer marked with black thick line [5].

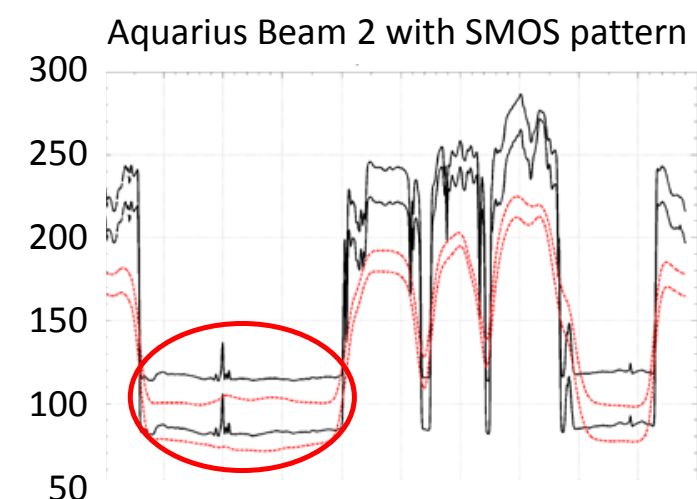

Time

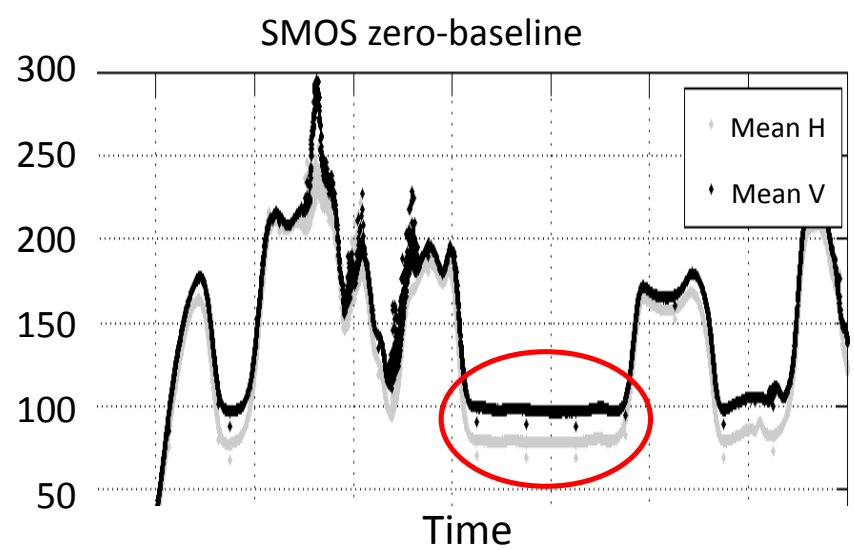

Time

Figure 2. Left: Comparison of simulated antenna temperature (vertical and horizontal polarization) when using Aquarius antenna pattern (black) and SMOS element antenna pattern (red) in Aquarius measurement geometry. Right: Measured antenna temperature of SMOS zero-baseline (following a sky maneuver) [5]. The results in the two plots are not concurrent and acquired at different incidence angles and pointing, but the overall level of the simulation with the SMOS antenna pattern and the actual zero-baseline measurement are very close.

\section{CONCLUSIONS}

The presentation will show the full 1.5-year simulation of SMOS zero-baselines compared with the actual measurements. The similarities and differences will be analyzed and the implications on the consistency of the brightness temperatures of SMOS and Aquarius will be assessed. Although the comparison is not done at the retrieval resolution of SSS, the results will give important direction where corrections are potentially required for building a continuous SSS product between the missions. 


\section{ACKNOWLEDGEMENTS}

The research described in this publication was carried out in part at the Jet Propulsion Laboratory, California Institute of Technology, under a contract with the National Aeronautics and Space Administration. The SMOS data was provided by the European Space Agency.

\section{REFERENCES}

[1] Y. Kerr et al., The SMOS Mission: New Tool for Monitoring Key Elements of the Global Water Cycle, Proc. of IEEE, Vol. 98, No. 5, May 2010.

[2] D. Levine, G. Lagerloef, S. Torrusio, Aquarius and Remote Sensing of Sea Surface Salinity from Space, Proc. of IEEE, Vol. 98, No. 5, May 2010.

[3] A. Colliander, L. Ruokokoski, J. Suomela, K. Veijola,J. Kettunen, V. Kangas, A. Aalto, M. Levander, H. Greus, M. Hallikainen, J. Lahtinen, Development and Calibration of SMOS Reference Radiometer, IEEE Trans. Geosci. Rem. Sens., Vol. 45, No. 7, July 2007.

[4] D. Le Vine, E. Dinnat, S. Abraham, P. de Matthaeis, F. Wentz, The Aquarius Simulator and Cold-Sky Calibration, IEEE Trans. Gesci. Rem. Sens., Vol. 49, No. 9, September 2011.

[5] J. Kainulainen, A. Colliander, J. Closa, M. Martin-Neira, R. Oliva, G. Buenadicha, P. Rubiales, A. Hakkarainen, M. Hallikainen, Radiometric Performance of the SMOS Reference Radiometers - Assessment after One Year of Operation, IEEE Trans. Gesci. Rem. Sens., In Press. 